\title{
Ein Implantatmaterial erobert den Markt
}

Roxolid, eine Metalllegierung aus Titan und Zirkonium, verfügt über eine bis zu 40\% höhere Ermüdungsfestigkeit gegenüber entsprechenden Titan-Implantaten (Bernhard et al., 2009) und ist auch in puncto Biokompatibilität überlegen. Die spezifischen Materialeigenschaften kommen insbesondere beim Einsatz von durchmesserreduzierten und den neuen kurzen $4 \mathrm{~mm}$-Implantaten von Straumann zum Tragen. Eine klinische Studie (Benic et al., 2013) belegt die Gleichwertigkeit von 3,3 mm-Roxolid-Implantaten gegenüber $\quad 4,1$ mm-Titanimplantaten. Dies ergibt neue Möglichkeiten für eine weniger invasive Behandlung von Patienten mit eingeschränktem Knochenangebot und der Vermeidung von aufwendigen und teuren Knochenaufbauten. Seit Jahresanfang 2014 bietet Straumann ein kostenloses Upgrade von Titan auf Roxolid.

Nach einer Pressemitteilung der Straumann GmbH, Freiburg Internet: www.straumann.de 